\title{
BDSMR: Velcro as a Sensory Material and Erotic Interface
}

\author{
Nolan Lem \\ Center for Computer Research in Music and Acoustics (CCRMA) \\ Stanford University \\ Stanford, USA \\ nlem@ccrma.stanford.edu
}

\begin{abstract}
This paper examines the sensory mechanics of the Velcro hook-and-loop fastener and its use as a sound object in contemporary sound-based art and the autonomous sensory meridian response (ASMR) community. With the neologism, BDSMR, I attempt to unpack the notion of velcro as a sound-fetish object by situating it within an emerging audio-visual culture, one that engages in cyborgian practices of sensory stimulation and optimizable arousal. Exploring these new modes of "listening in on" materials-oftentimes with disembodied binaural ears-we reveal the ways in which the dynamics of the sound object reorientate our understanding of the sensate body and its relationship to the eroticism of aurality. In doing so, I present my own Velcro-based sound sculptures in an attempt to form a dialogue with the velcro material as an artistic medium.
\end{abstract}

Aurality, Eroticism, Cyborg, ASMR, BDSM, Sound Studies, Sound Art, Contemporary Art.

\section{ASMR}

Autonomous Sensory Meridian Response (ASMR) is an evoked physiological response to specific types of audio-visual stimuli. Sometimes referred to as an auditory-tactile synaesthesia, the most common descriptions of ASMR refer to the feeling as a brain tingle or orgasm. For the most part, ASMR communities inhabit online spaces where users share videos that are associated with a dizzying array of ASMR-related content (as shown in Figure 1), many of them meta-tagged using a combination of semantic descriptors that allow the user to filter the content they seek.

Taken as a whole, the ASMR sound world is vast, understudied and enigmatic. However, typical ASMR sounds tend to be quiet, sparse, nonrepetitive, context-specific and non-pitched where everyday household items (brushes, paper, plastic sheets, ball-point pens etc.) are oftentimes used as performative instruments to gauge the sonic potential of a material. [intentional], [unintentional], [roleplay], [male],[female], [whisper], [softspoken],[unintelligible], [no talking], [mouth sounds], [eating] [French accent], [Russian accent], [Korean], [polish], [roleplay], [brushing], [tapping], [crinkling], [scratching], [woo], [latex gloves], [close up], [short], [no props], [clicking], [visual asmr], [hand movements], [trigger words], [tongue clicking], [pen light], [face measuring], [skin inspection], [whispered], [Portuguese], [make-up application], [no talking], [animal], [eating], [chewing], [girlfriend roleplay], [burger eating sounds], [nature sounds], [soft spoken], [ambient sound], [slime], [mic test], [Velcro], [British], [Gaming], [Relaxing], [Controller tapping], [satisfying], [wind], [phone typing], [glass sounds], [liquid sounds], [Stippling], [Pencil sounds], [weird], [binaural], [fruit], [cuddly toys], [collab], [calligraphy], [Italian Accent], [vampire], [boyfriend roleplay], [amateur], [ear massage], [slurping], [sipping], [gulping], [game talk], [ramble], [asmr news], [ear to ear], [pet therapy], [caressing], [fast scratching], [slow scratching], [art], [draw], [cooking sounds], [skittles], [sand cutting], [show and tell], [irish], [birds], [piano], [page turning], [writing sounds], [indian accent], [candy], [foley], [rhythmic], [fire crackling], [nail tapping], [cute], [tiny toys], etc..

Figure 1: Metatags of common ASMR videos

Velcro sounds are but a tiny sliver of the audio-visual content in the ASMR community. Here we see the tagging of videos as a means through which users not only seek to understand their own sensory response but as a means through which they aim to optimize it. In this feedback loop, the production and consumption of ASMR videos operate as a sort of iterative heuristic through which userscrowd-source a video's evocative potential. A more quantitative analysis of the ASMR community might oversee the construction of an "actor-network map" which would be crucial for reassembling the ways in which these categories emerge, disseminate and evolve over time. In general, the sound objects operate as 
actants in a network that facilitate heightened states of arousal and attention between object and performer where a tentative intimacy is rendered between performer and listener, listener and body. These sensory instruments provide a way for users to probe the sensate body to register new modes of situational awareness and therapy.

\subsection{To Be or Not To Be Attached: BANDS WIDTH}

To perform with velcro is to engage with it as a rigid material where we emancipate the frictive energies bound up in its hooks and loops. However, the struggle of unfurling a velcro strip is a feature not a bug; it is precisely this agonistic quality in the material that facilitates the trance or "flow-like" state often accompanying the sensory meridian response (Barratt, 2015). Of course, as a "stochastic" adhesive-as a material governed by statistical design - velcro was engineered with its probabilistic mechanics in mind. Referring to the original patent illustrations in Figure 2, each hook and loop, when presented in spatial proximity, take part in an interactive game: the probability of the hook becoming attached to the loop is great enough so that taken as a whole, the tensile strength of the successful hook-looped bonds allow it to bear a structural load. The process through which these fibres become extricated sum, fibre by fibre, to synthesize its distinctive ripping sound.

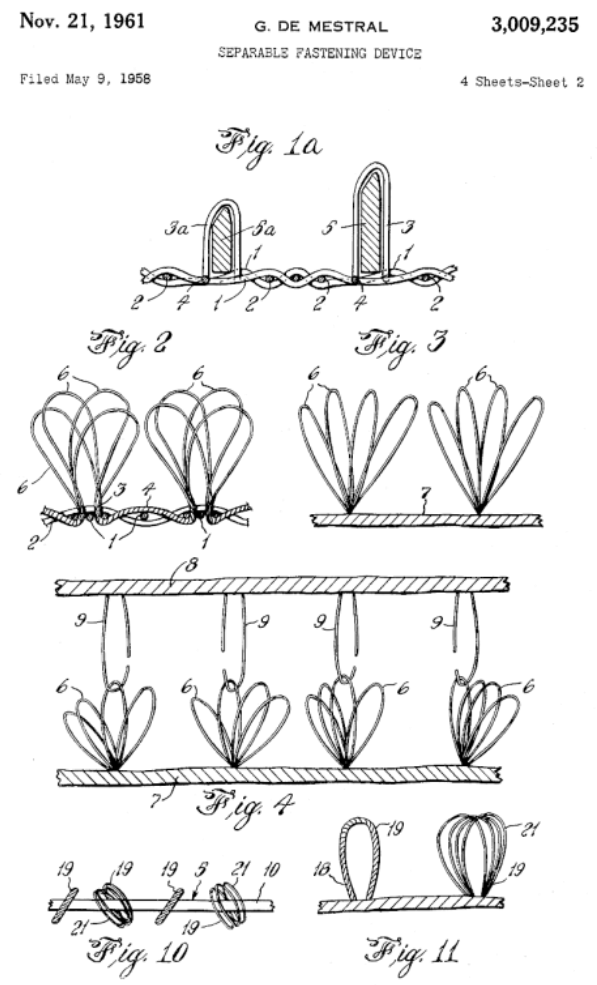

Figure 2: Original Patent Illustration for Separable Fastening Device (1961, Mestral)
One way to model the velcro sound statistically is by treating the outcomes of the hook and loop events as a discrete random variable within a Poisson distribution. This type of distribution is useful for modelling events that occur independently of one another in time and with a constant rate. This model is useful insofar as it grounds the structure of my first velcro-based sculpture, BANDS WIDTH (2016), which features two kinetic machines that rotate cylindrical rollers surfaced with 192 sq. inches of velcro-on-velcro contact. The machines are themselves are turned on and off according to a Poisson distribution with a mean time of one minute. Here, we have the probabilistic mechanics of velcro playing out in two different temporal levels: at the level of the individual hook and loop and at the level of the machines being activated. This type of recursion features prominently in the work insofar as different probability functions control the behaviour of the piece at both the microscale-the connections of between hooks and loops-and the macroscalethe probability that a machine will be turned on. Standing in front of the machines, one is confronted with the rich timbral density of velcro in a perpetual ripping-apart, which at times presents itself as sentient and ethereal.

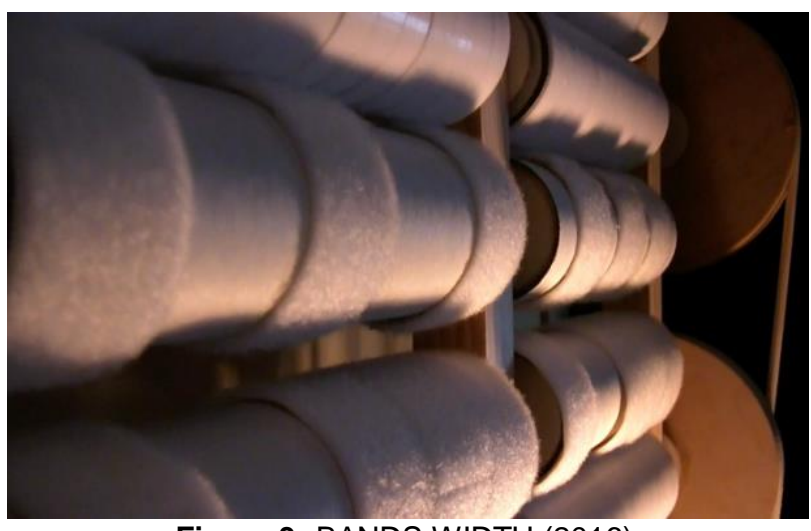

Figure 3: BANDS WIDTH (2016)

In this piece, I wanted to survey the strange acoustic space actualized by the velcro material by using the machines to traverse the boundaries of silence and noise. Somewhere in between these shifting textures, we find the sensory sweet spot shared by those in the velcrophile community.

\subsection{Exploratory Objects and Devices}

In "Naked Intimacy: Eroticism, Improvisation, and Gender", Ellen Waterman defines improvisation as a "performative mode that brings the musician into an erotic encounter with the present moment (an ecstatic communication with and through the self, others, instrument, environment). To improvise is to give oneself up entirely (luxuriously, generously) to the now over and over again" (Waterman, 2008). 
Improvisation is intimately bound up with the passage of time and the awareness of its passing. In the situational intimacy rendered by ASMR, the performance of listening and feeling the sustaining power of the ASMR redefines the now over and over again. Not unlike the use of fetish objects in BDSM, the use of Velcro in ASMR videos can be viewed as an exploratory device that that elicits, teases and probes a range of physiological reactions each reasserting the erotic encounter in the present moment. In this "ecstatic communication" with the sensate body, we reveal the latent articulations of the transgressive desire bound up in our unknowing. This representation of sound-as-fetish is literally front and center in my sound sculpture, autonomous sense object (2017) which sees an appendage shrouded in leather bondage being pecked, probed and poked by a kinetic array of prosthetic models of my own Velcro-tipped fingers. Speakers are driven acoustically by velcro extrications that transfer energy into the paper speaker cones where they amplify the velcro ripping sound at the base of the sculpture. Casting a physical body as a cyborgian object of erotic materiality, this piece makes explicit the connection between sensory organ, aural fetish and the automation of desire. The ceaselessness of the gestures-the rotation of the leathery velcroladen object, the compulsions of the fingers prodding-point to the ways in which the ASMR audience is bound to the sound object like a slave bound to a master in certain practices of BDSM.

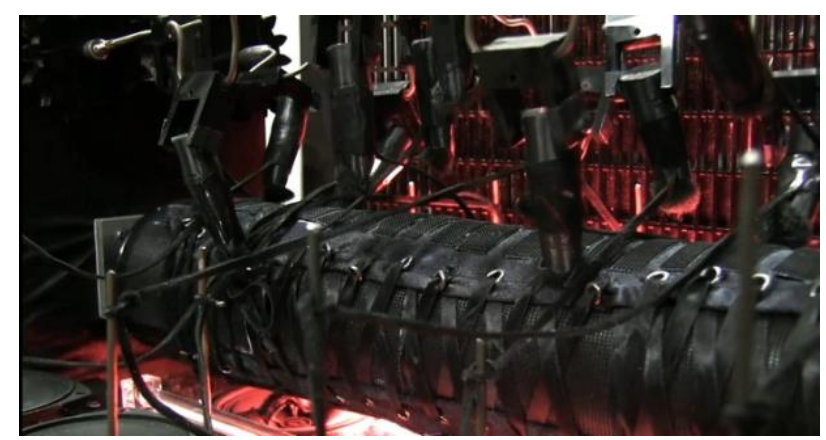

Figure 4: Autonomous Sense Object (2016)

Tentacule (2018) is another sound machine that rotates a large Velcro-ribbed object like a piece of roasting meat on a barbeque spit. Using the same excitation mechanism as 'autonomous sense object', the base of the sculpture houses ten speakers which are acoustically driven by velcro extrication that occur alongside the point of contact between object and speaker. In this case, the dynamics of the velcro becoming hooked and unfastened are transmitted through large plastic "tentacules" that resonate and transfer velcro ripping sounds into different parts of an acoustic space. The imposing cephalopodic presence of the black machine suggests a cyborgian instrument somewhere in between an organ, a music box and a Luigi Russolo noise machine (Russolo, 1916).

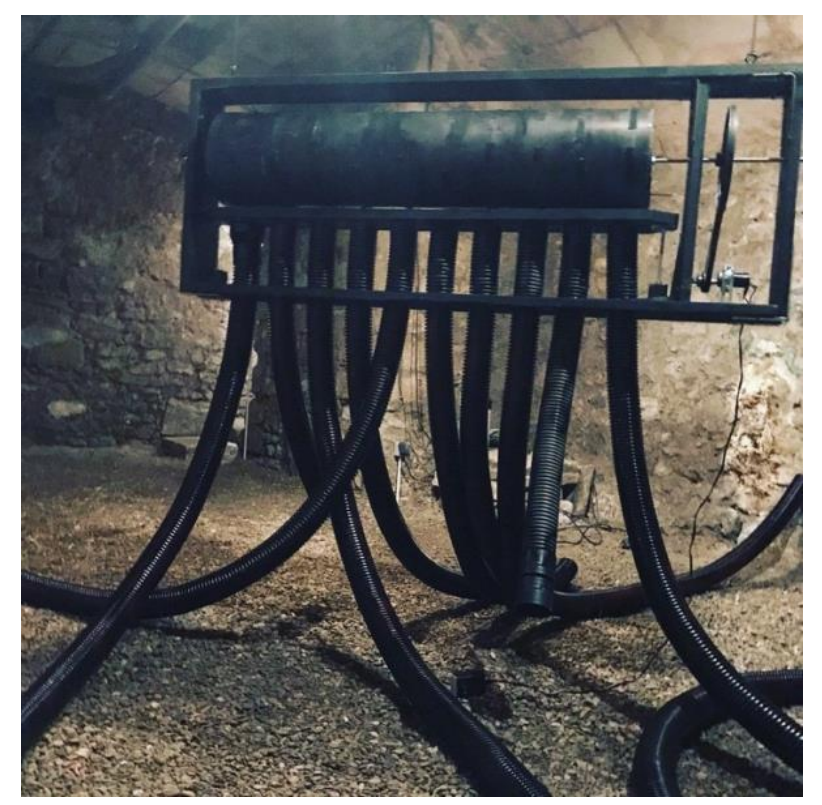

Figure 5: Tentacule (2018)

The work l've presented here is a testament to the ways in which the ASMR community is impacting others far and wide. These pieces have enabledme to access my own relationship with sound and sensory experience as embodied by the design of these "emotional" machines. As the content of ASMR changes to accommodate different cultural landscapes, we too will have to update our understanding of sound, fetish, and materiality within ASMR.

\section{REFERENCES}

Barratt, E., Davis, N. (2015) Autonomous Sensory Meridian Response (ASMR): a flow-like mental state. PeerJ, 3: e851.

Copeland, L. (2017) How Researchers Are Beginning to Gently Probe the Science Behind ASMR. https://www.smithsonianmag.com/sciencenature/researchers-begin-gently-probe-sciencebehind-asmr-180962550/ (15 June 2018)

Mestral, G. (1961) Separable Fastening Device. US3009235. US Patent Office

Russolo, L., Lanuzza, S. (1916) L'arte dei rumori. Edizioni futuriste di Poesia.

Waterman, E. (2008) Naked Intimacy: Eroticism, Improvisation, and Gender. Critical Studies in Improvisation / Études critique en improvisation. 4. 10.21083/csieci.v4i2.845 